\title{
Binding Energies in Nonrelativistic Field Theories
}

hep-lat/9608139

Andreas S. Kronfeld ${ }^{\mathrm{a} *}$

FERMILAB-CONF-96/237-T

a Theoretical Physics Group, Fermi National Accelerator Laboratory, Batavia, Illinois, USA

Relativistic corrections communicate the binding energy of a bound state to its kinetic mass. This mechanism is reviewed and used to explain anomalous results of Collins, Edwards, Heller, and Sloan (hep-lat/9512026), which compared rest and kinetic masses of heavy-light mesons and quarkonia.

\section{INTRODUCTION}

Last year Collins, Edwards, Heller, and Sloan [1] studied heavy Wilson quarks [2] with the improved action of Sheikholeslami and Wohlert [3]. In lattice units the heavy-quark mass $m_{Q} a$ typically exceeded unity, a regime in which the numerical results require a nonrelativistic interpretation [4.5] just as in NRQCD [6, [7. Ref. [1] presents a test of the nonrelativistic interpretation that removes kinematic effects and focuses on a dynamical effect - the binding energy. The results of this test were unexpectedly anomalous. The aim of this paper is to explain why and to offer a remedy.

Let us begin with some basics to define notation. As a function of momentum $\boldsymbol{p}$ the energy of a state $X$ can be written

$E_{X}(\boldsymbol{p})=M_{1 X}+\frac{\boldsymbol{p}^{2}}{2 M_{2 X}}-\frac{\left(\boldsymbol{p}^{2}\right)^{2}}{8 M_{4 X}^{3}}+\cdots$,

where the rest mass is defined by $M_{1}=E(\mathbf{0})$, and the kinetic mass is defined by

$M_{2}=\left(\frac{\partial^{2} E}{\partial p_{i}^{2}}\right)_{\boldsymbol{p}=\mathbf{0}}^{-1}$.

Below the states can be quarks, $Q$ and $q$, and mesons $\bar{Q} Q, \bar{Q} q$, and $\bar{q} q$. Usually $Q$ is assumed heavier than $q$.

On a relativistic mass shell

$M_{1 X}=M_{2 X}=\cdots=m_{X}$,

In this paper the lower-case $m_{X}$ denotes the exact, physical mass, whereas upper-case $M_{i X}$ de-

*Poster given at Lattice '96, 4-8 June 1996, St. Louis, Missouri note the result of a (possibly approximate) calculation. In the mass-dependent renormalization of ref. [5] it is possible to adjust the action's couplings to recover eq. (3), to a specified accuracy. With the Wilson or Sheikholeslami-Wohlert actions, however, perturbation theory shows that $M_{1 Q} \neq M_{2 Q}$ (except as $\left.m_{Q} a \rightarrow 0\right)$. In nonrelativistic systems this is acceptable, provided one adjusts the bare mass until the kinetic mass takes its physical value [4,5], just as in ref. [6,7].

The quark state makes sense at most in perturbation theory. In a nonperturbative world, one would like to carry out the tuning with a bound state, e.g. a meson $X=\bar{Q} q$ whose masses $M_{1 \bar{Q} q}$, $M_{2 \bar{Q} q}$, etc, can be computed with Monte Carlo methods. Let us define the binding energy $B$ by

$$
\begin{aligned}
& M_{1 \bar{Q} q}=M_{1 \bar{Q}}+M_{1 q}+B_{1}, \\
& M_{2 \bar{Q} q}=M_{2 \bar{Q}}+M_{2 q}+B_{2} .
\end{aligned}
$$

To make a precise definition of the binding energies, one requires a precise definition of the quark masses in eq. (4). Here it is enough to take the rest and kinetic masses of the free theory $\left(g_{0}^{2}=0\right)$ with the same bare quark masses. Computing bound-state splittings through rest masses makes sense only if $B_{1}$ is the experimental binding energy, to sufficient accuracy. Similarly, tuning the meson mass to the kinetic mass makes sense only if also $B_{2}$ is the experimental binding energy, to sufficient accuracy.

As shown below, the anomalous result of ref. [1] is sensitive to $B_{2}-B_{1}$. There are two keys to understanding it. First, one must be careful about the qualifying phrase "to sufficient accuracy" in the preceding paragraph. Second, one must to 
know how field theories communicate the binding energy to a bound state's kinetic mass.

Sect. 2 recalls the diagnostic test of ref. [1]. Sect. 3 assesses the cutoff effects of the binding energy in light-light and heavy-light mesons, and in quarkonium. The mechanism for generating the "kinetic binding energy" is reviewed for a relativistic (continuum) gauge theory in sect. A and generalized to lattice gauge theory in sect. 5 . Sect. 6 draws a few conclusions.

\section{THE TEST}

Let us abbreviate $\delta M:=M_{2}-M_{1}$ and $\delta B:=$ $B_{2}-B_{1}$. Ref. [1] introduces

$I:=\frac{2 \delta M_{\bar{Q} q}-\left(\delta M_{\bar{Q} Q}+\delta M_{\bar{q} q}\right)}{2 M_{2 \bar{Q} q}}$.

Comparison with eqs. (4) shows that the quark masses drop out, leaving

$I=\frac{2 \delta B_{\bar{Q} q}-\left(\delta B_{\bar{Q} Q}+\delta B_{\bar{q} q}\right)}{2 M_{2 \bar{Q} q}}$.

If the lattice action(s) of the quarks were sufficiently accurate, all $\delta B \mathrm{~s}$, and hence $I$, would vanish. ( $I$ vanishes trivially when $m_{\bar{Q}}=m_{q}$, even if $\delta B_{\bar{Q} q} \neq 0$.)

The numerical results of ref. [1] are shown in fig. 11. The "inconsistency" $I$ is negative, and $|I|$ tends to increase with increasing $m_{Q}$. To explain both the sign and the magnitude, below I shall derive an expression for $\delta B$.

\section{CUTOFF EFFECTS ON $\delta B$}

Before presenting the analytical result for $\delta B$, it is useful to anticipate the order of magnitude of $\delta B$ in each meson - light-light, heavy-light, and quarkonium. On this basis it turns out that the quarkonium $\delta B_{\bar{Q} Q}$ dominates the numerator in eq. (6).

\subsection{Light-light $\delta B_{\bar{q} q}$}

The binding energy is $\mathrm{O}\left(\Lambda_{\mathrm{QCD}}\right)$. With the Sheikholeslami-Wohlert action, $B_{1}$ and $B_{2}$ both suffer from lattice artifacts of $\mathrm{O}\left(\alpha^{n} a \Lambda_{\mathrm{QCD}}\right)$. With the tree-level improvement of used by ref. [1], $n=1$. (With the Wilson action $n=0$.)

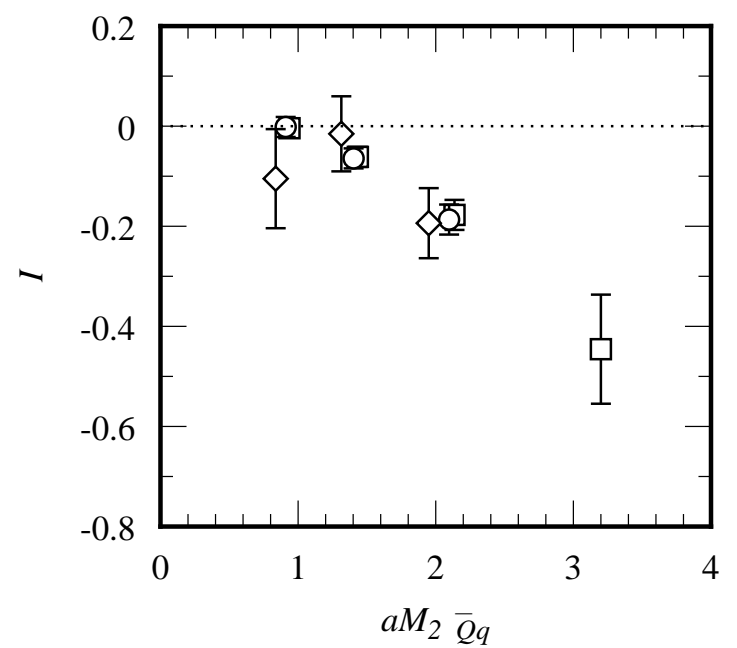

Figure 1. Plot of the binding-energy "inconsistency" $I$ vs. the heavy-light meson's kinetic mass $a M_{2 \bar{Q} q}$, for $m_{Q} \gg m_{q}$. Adapted from ref. [1].

There is no reason for the artifacts to be identical, so $\delta B_{\bar{q} q}$ is $\mathrm{O}\left(\alpha^{n} a \Lambda_{\mathrm{QCD}}^{2}\right)$. This is numerically small, so $\delta B_{\bar{q} q}$ can be neglected below.

\subsection{Heavy-light $\delta B_{\bar{Q} q}$}

The binding energy is again $\mathrm{O}\left(\Lambda_{\mathrm{QCD}}\right)$. The light quark suffers lattice artifacts as above, but, when $m_{Q} \gtrsim 1$ - as in fig. 1, the heavy quark also suffers from (smaller) effects of $\mathrm{O}\left(\Lambda_{\mathrm{QCD}}^{2} a / m_{Q}\right)$. Again, even though there is no reason for artifacts in $B_{1}$ and $B_{2}$ to cancel, one sees that $\delta B_{\bar{Q} q}$ is numerically negligible.

\subsection{Quarkonium $\delta B_{\bar{Q} Q}$}

The binding energy is now $\mathrm{O}\left(m_{Q} v^{2}\right)$, where $v$ denotes the relative $\bar{Q}-Q$ velocity. In this nonrelativistic system, the velocity is a pertinent estimator of cutoff effects [7,5]. The rest mass is $\mathrm{O}\left(v^{0}\right)$, so the action would need absolute accuracy of $\mathrm{O}\left(v^{2}\right)$ to obtain relative accuracy of $\mathrm{O}\left(v^{2}\right)$ in $B_{1}$. Both the Wilson and Sheikholeslami-Wohlert actions achieve this. On the other hand, the kinetic mass multiplies an $\mathrm{O}\left(v^{2}\right)$ effect, so the action would now need an absolute accuracy of $\mathrm{O}\left(v^{4}\right)$ to obtain relative accuracy of $\mathrm{O}\left(v^{2}\right)$ in $B_{2}$. Neither the Wilson nor the Sheikholeslami-Wohlert action achieves this [5]; with either of them, one can only 
hope for $\mathrm{O}\left(v^{0}\right)$ relative accuracy in $B_{2}$. The error in $B_{2}$, and hence in $\delta B_{\bar{Q} Q} \neq 0$, is $\mathrm{O}\left(m_{Q} v^{2}\right)$, which is significantly larger than the previous two estimates.

\section{BREIT EQUATION}

For nonrelativistic systems the binding-energy discrepancy can be worked out quantitatively, following a textbook nonrelativistic expansion of QED [8]. This section verifies in a relativistically invariant theory that $B_{2}=B_{1}=B$. The next section then turns to the lattice theories, which break relativistic invariance. For convenience, these two section assume that even the "light" quark $q$ is nonrelativistic.

At leading order the quark-anti-quark interaction arises from one-gluon exchange diagrams. Evaluating these diagrams and developing the nonrelativistic expansion, one obtains a Hamiltonian $H=m_{\bar{Q}}+m_{q}+H_{2}+H_{4}$ for the quarkanti-quark system. The leading nonrelativistic dynamics are given by

$$
\begin{aligned}
H_{2} & =\frac{\boldsymbol{p}_{\bar{Q}}^{2}}{2 m_{\bar{Q}}}+\frac{\boldsymbol{p}_{q}^{2}}{2 m_{q}}+V(r) \\
& =\frac{\boldsymbol{P}^{2}}{2 M_{\bar{Q} q}}+\frac{\boldsymbol{p}^{2}}{2 \mu}+V(r),
\end{aligned}
$$

where $V(r)=-C_{F} \alpha / r ; \boldsymbol{r}=\boldsymbol{x}_{\bar{Q}}-\boldsymbol{x}_{q}, \boldsymbol{P}$ and $\boldsymbol{p}$ are center-of mass coordinates and momentum; $\mu=\left(m_{\bar{Q}}^{-1}+m_{q}^{-1}\right)^{-1}$ is the reduced mass; and $M_{\bar{Q} q}=m_{\bar{Q}}+m_{q}$. The first relativistic corrections are

$H_{4}=-\frac{\left(\boldsymbol{p}_{\bar{Q}}^{2}\right)^{2}}{8 m_{\bar{Q}}^{3}}-\frac{\left(\boldsymbol{p}_{q}^{2}\right)^{2}}{8 m_{q}^{3}}+V_{2}\left(\boldsymbol{r} ; \boldsymbol{p}_{Q}, \boldsymbol{p}_{q}\right)$,

where the non-local potential $V_{2}$ is given by Breit's equation [8]. It takes the form

$$
\begin{aligned}
& V_{2}\left(\boldsymbol{r} ; \boldsymbol{p}_{Q}, \boldsymbol{p}_{q}\right)=\text { const } \times \delta^{(3)}(\boldsymbol{r}) \\
& \quad+V(r)\left[1-\frac{\boldsymbol{p}_{\bar{Q}} \cdot \boldsymbol{p}_{q}+r^{-2} r_{i} r_{j} p_{\bar{Q} i} p_{q j}}{2 M_{\bar{Q} q} \mu}\right] \\
& \quad+\text { spin-dependent. }
\end{aligned}
$$

The spin-dependent terms and the terms proportional to $\delta^{(3)}(\boldsymbol{r})$ are not important here. Full details are given in $\S \S 83-84$ of ref. [8]. Together with the $\left(\boldsymbol{p}^{2}\right)^{2}$ terms in $H_{4}$, the exhibited part of $V_{2}$ is responsible for modifying the bound-state kinetic mass from $M_{\bar{Q} q}=m_{\bar{Q}}+m_{q}$ to $m_{\bar{Q} q}=m_{\bar{Q}}+m_{q}+B$ (as required by Lorentz invariance).

To proceed one must re-write $H_{4}$ in center-ofmass momenta and collect terms quadratic in the total bound-state momentum $\boldsymbol{P}$. In the bound state, combinations of the internal momentum $\boldsymbol{p}$ and relative coordinate $\boldsymbol{r}$ can be replaced by expectation values. Collecting all terms, the boundstate kinetic energy becomes

$$
\begin{aligned}
\frac{\boldsymbol{P}^{2}}{2 m_{\bar{Q} q}} & :=\frac{\boldsymbol{P}^{2}}{2 M_{\bar{Q} q}}\left(1-\frac{\langle T+V\rangle}{M_{\bar{Q} q}}\right) \\
& +\frac{P_{i} P_{j}}{2 M_{\bar{Q} q}^{2}}\left\langle r_{i} \nabla_{j} V-p_{i} p_{j} / \mu\right\rangle,
\end{aligned}
$$

where $T=\boldsymbol{p}^{2} / 2 \mu$ is the internal kinetic energy. By the virial theorem the second line vanishes. Thus, to consistent order in $\boldsymbol{p} / M$ the leading relativistic corrections $H_{4}$ generates the right binding energy $B_{2}=\langle T+V\rangle=: B$ for the bound-state kinetic mass.

More generally, higher-order relativistic effects trickle down to bound-state properties as follows: the correction of $\mathrm{O}\left(v^{\ell}\right)$ provides the $\mathrm{O}\left(v^{\ell-k}\right)$ contribution to bound-state properties of $\mathrm{O}\left(v^{k}\right)$.

\section{LATTICE GENERALIZATION}

On a hypercubic lattice there can be two corrections to the kinetic energy

$E(\boldsymbol{p})=\cdots-\frac{\left(\boldsymbol{p}^{2}\right)^{2}}{8 M_{4}^{3}}-\frac{1}{6} w_{4} a^{3} \sum_{i} p_{i}^{4}+\cdots$,

for each of $\boldsymbol{p}=\boldsymbol{p}_{\bar{Q}}, \boldsymbol{p}_{q}$. Here

$M_{4}:=-\left(\frac{\partial^{4} E}{\partial p_{i}^{2} \partial p_{j}^{2}}\right)_{\boldsymbol{p}=\mathbf{0}}^{-1 / 3}, \quad i \neq j$

and

$w_{4}:=-\left.\frac{1}{4} \frac{\partial^{4} E}{\partial p_{i}^{4}}\right|_{\boldsymbol{p}=\mathbf{0}}-\frac{3}{4 M_{4}^{3}}$.

Unless the action has been improved further than the Sheikholeslami-Wohlert action, $M_{4} \neq M_{2}$ and 
$w_{4} \neq 0$, cf. Appendix A of ref. [5]. These lattice artifacts filter through to $B_{2}$ - just as abovethrough the terms proportional to $P_{i} P_{j}\left\langle p_{i} p_{j}\right\rangle$.

The spatial gluon generates the spinindependent contribution proportional to $V(r)$ in eq. (9); on the lattice the nuance is that the kinetic mass appears in the bracket. On the other hand, the temporal gluon generates more complicated terms, but they either depend on spin or are proportional to $\delta^{(3)}(\boldsymbol{r})$. So to work out an expression for $B_{2}$, it is enough to maintain eq. (9), but with masses $M_{2 \bar{Q} q}$ and $\mu_{2}$ built from $M_{2 \bar{Q}}$ and $M_{2 q}$.

The calculation of the binding energy difference $\delta B$ follows the steps leading to eq. (10). One finds an expression that is too cumbersome to present here. In an $\mathrm{S}$ wave, however, it can be simplified because $\left\langle p_{i} p_{j}\right\rangle=\frac{1}{3} \delta_{i j}\left\langle p^{2}\right\rangle$. Then

$$
\begin{aligned}
\frac{\delta B}{\langle T\rangle}=\frac{1}{3} & \left\{5\left[\mu_{2}\left(\frac{M_{2 \bar{Q}}^{2}}{M_{4 \bar{Q}}^{3}}+\frac{M_{2 q}^{2}}{M_{4 q}^{3}}\right)-1\right]\right. \\
& \left.+4 a^{2} \mu_{2}\left(M_{2 \bar{Q}}^{2} w_{4 \bar{Q}}+M_{2 q}^{2} w_{4 q}\right)\right\} .
\end{aligned}
$$

This is the main new result of this paper. Note that, as one would have anticipated, the expression vanishes when $w_{4 X}=0$ and $M_{4 X}=M_{2 X}$.

With an estimate of $\langle T\rangle$ from potential models [9] and the lattice masses of the right-most point in fig. 1, I find

$I \approx-\frac{\delta B_{\bar{Q} Q}}{2 M_{\bar{Q} q}} \approx-0.5$.

The agreement with the Monte Carlo results is surprisingly good.

\section{CONCLUSIONS}

The origin of the anomaly observed in ref. [1] is the usage of an action accurate only to $\mathrm{O}\left(v^{2}\right)$. Thus the relative error in the binding energy $B_{2}$ of the bound-state kinetic mass is of order $m_{Q} v^{2} / m_{Q} v^{2}=1$. Meanwhile, the usual binding energy $B_{1}$ is indeed valid to leading order in $v^{2}$. The test quantity $I$ cleverly isolates $B_{2}-B_{1}$, and thus exposes an inconsistency of $\mathrm{O}(1)$.
By examining how (approximately) relativistic field theories generate $B_{2}$, this paper explains the results found last year [1]. Moreover, the analysis makes the remedy plain: the anomaly is not expected to appear if quarkonium properties are computed with an action improved through $\mathrm{O}\left(v^{4}\right)$ (or higher). In particular, one requires $M_{4}=M_{2}$ and $w_{4}=0$.

Most published applications of ref. $\sqrt{6} \|$ use a sufficiently accurate action [7]. Ref. [10] even remarks that $\mathrm{O}\left(v^{4}\right)$ accuracy is essential for a consistent determination of the $b$-quark mass from the $\Upsilon$ spectrum. For four-component fermions the details required for $\mathrm{O}\left(v^{4}\right)$ accuracy in quarkonium have appeared more recently [5].

\section{ACKNOWLEDGEMENTS}

I would like to thank Sara Collins and John Sloan for bringing their results to my attention and for insisting that I explain it. Fermilab is operated by Universities Research Association, Inc., for the U.S. Department of Energy.

\section{REFERENCES}

1. S. Collins, R. Edwards, U. Heller, and J. Sloan, hep-lat/9512026, Nucl. Phys. B Proc. Suppl. 47 (1996) 455.

2. K.G. Wilson, in New Phenomena in Subnuclear Physics, A. Zichichi (ed.), Plenum, New York, 1977.

3. B. Sheikholeslami and R. Wohlert, Nucl. Phys. B259 (1985) 572.

4. P.B. Mackenzie, Nucl. Phys. B Proc. Suppl. 30 (1993) 35;

A.S. Kronfeld, Nucl. Phys. B Proc. Suppl. 30 (1993) 445, 42 (1995) 415.

5. A.X. El-Khadra, A.S. Kronfeld, and P.B. Mackenzie, hep-lat/9604004, FERMILABPUB-96/074-T, ILL-TH-96-1.

6. G.P. Lepage and B.A. Thacker, Nucl. Phys. B Proc. Suppl. 4 (1987) 199;

B.A. Thacker and G.P. Lepage, Phys. Rev. D43 (1991) 196.

7. G.P Lepage, L. Magnea, C. Nakhleh, U. Magnea, and K. Hornbostel, Phys. Rev. D46 (1992) 4052. 
8. V.I. Berestetskii, E.M. Lifshitz, and L.P. Pitaevskii, Relativistic Quantum Theory, Pergamon, Oxford, 1971.

9. A.X. El-Khadra, private communication.

10. C.T.H. Davies et al, Phys. Rev. Lett. 73 (1994) 2654. 